\title{
DNA-Templated Three-Branched Nanostructures for Nanoelectronic Devices
}

Héctor A. Becerril, Randall M. Stoltenberg, Dean R. Wheeler, Robert C. Davis, John N. Harb, and Adam T. Woolley

\section{Supporting Information}

\section{Oligonucleotide design}

Oligonucleotides 1, 2 and 3 are 125-, 125- and 118-mers, respectively; each has an extended length of $\sim 40 \mathrm{~nm}$ and a melting temperature of $\sim 74{ }^{\circ} \mathrm{C}$. Oligonucleotide 4 is a 40 -base segment with a melting temperature of $\sim 52{ }^{\circ} \mathrm{C}$ and a biotin attached to position 20. Oligonucleotides 1-3 were selected and analyzed for primer-dimer complexes using software written in house. They were further analyzed for hairpins using the DNA folding and energy minimization algorithm implemented in RNAstructure. ${ }^{1}$ The sequences of the oligonucleotides used in this work are given below. Table 1 summarizes the findings on the maximum lengths and melting temperatures $\left(\mathrm{T}_{\mathrm{m}}\right)$ of the primer-dimer and hairpin complexes for oligonucleotides $\mathbf{1}, \mathbf{2}$ and $\mathbf{3}$.

Oligonucleotide 1:

5'GGGCGGCGACCTGCTCATCAGCTACCGGCTACCATTCGGCGCTAGAAAAAAAAAA AAAAAAAAAAAAAAAAAAAAAAAAAAAAAACCGTGTCACAAGGTGCATCGTAACC TCACATTGCGGGGGT3'

Oligonucleotide 2:

5'GGGCGGCGACCTACCCCCGCAATGTGAGGTTACGATGCACCTTGTGACACGGAAA AAAAAAAAAAAAAAAAAAAAAAAAAAAAAAAAAAAAAGTACAGACAACAGGCAC GACGCACTTCTCGGAG3'

Oligonucleotide 3:

5'GGGCGGCGACCTCTCCGAGAAGTGCGTCGTGCCTGTTGTCTGTACAAAAAAAAAA AAAAAAAAAAAAAAAAAAAAAAAAAAAAAACTAGCGCCGAATGGTAGCCGGTAGC TGATGAGC3'

Oligonucleotide 4:

5’TTTTTTTTTTTTTTTTTTT[BioTEG]TTTTTTTTTTTTTTTTTTTT3’

Table 1. Primer-dimer and hairpin complexes in designed oligonucleotide sequences

\begin{tabular}{|c|c|r|c|c|c|c|}
\hline Oligonucleotide & $\begin{array}{l}\text { Longest primer- } \\
\text { dimer complex (base } \\
\text { pairs) }\end{array}$ & Count & $\begin{array}{l}\text { Maximum primer- } \\
\text { dimer } \mathrm{T}_{\mathrm{m}}\left({ }^{\circ} \mathrm{C}\right)\end{array}$ & $\begin{array}{l}\text { Longest } \\
\text { hairpin (base } \\
\text { pairs) }\end{array}$ & Count & $\begin{array}{l}\text { Maximum } \\
\text { hairpin } \mathrm{T}_{\mathrm{m}}\left({ }^{\circ} \mathrm{C}\right)\end{array}$ \\
\hline $\mathbf{1}$ & 4 & 9 & 0.7 & 3 & 6 & -3.3 \\
\hline $\mathbf{2}$ & 4 & 12 & 0.7 & 4 & 1 & 0.7 \\
\hline $\mathbf{3}$ & 6 & 1 & 8.7 & 5 & 1 & 4.7 \\
\hline
\end{tabular}




\section{DNA complex assembly}

Oligonucleotides (Qiagen, Valencia, CA) were aliquoted in Tris-EDTA (TE) buffer (pH 8.0) to a concentration of $10 \mathrm{nM}$. Equal volumes of oligonucleotides 1, 2 and 3 were mixed with $1 / 2$ volume of $10 \mathrm{X}$ annealing buffer (Qiagen) in a microcentrifuge tube, heated to $95{ }^{\circ} \mathrm{C}$ for $2 \mathrm{~min}$ and allowed to cool to room temperature. A four-fold stoichiometric excess of oligonucleotide 4, a two-fold stoichiometric excess of streptavidin, and sufficient $\mathrm{NaCl}$ to provide a final concentration of $500 \mathrm{mM} \mathrm{Na}^{+}$were added to the tube. Then, the mixture was heated to $60{ }^{\circ} \mathrm{C}$ for $10 \mathrm{~min}$ and allowed to cool to room temperature.

\section{Atomic force microscopy}

Data were collected with a Multimode IIIa AFM (Veeco, Sunnyvale, CA) using microfabricated, aluminum-coated silicon cantilever tips (Nanoscience Instruments, Phoenix, AZ). Vibrational noise was reduced with an active isolation system (MOD1-M, Halcyonics, Goettingen, Germany). Representative imaging parameters were: tip resonance frequency, $50-60 \mathrm{kHz}$; free oscillation amplitude, 0.9-1.1 V; amplitude setpoint, 0.5-0.8 V; scan rate, 1.0-1.6 Hz. Images were processed offline to remove background curvature.

\section{Preparation of surfaces for microscopy analysis}

Freshly cleaved mica surfaces were treated with a $1 \mathrm{ppm}$ aqueous solution of poly-L-lysine (Ted Pella, Redding, CA) for $10 \mathrm{~min}$, rinsed with water and air dried. Carbon-coated copper TEM grids were treated with $5 \mathrm{ppm}$ aqueous poly-L-lysine for $10 \mathrm{~min}$, rinsed with water and dried. Complexes $\mathbf{A}$ and $\mathbf{B}$ were deposited on both types of surfaces immediately after drying. Figure S-1 shows typical overview and zoomed images of deposited DNA complexes $\mathbf{A}$ and $\mathbf{B}$.

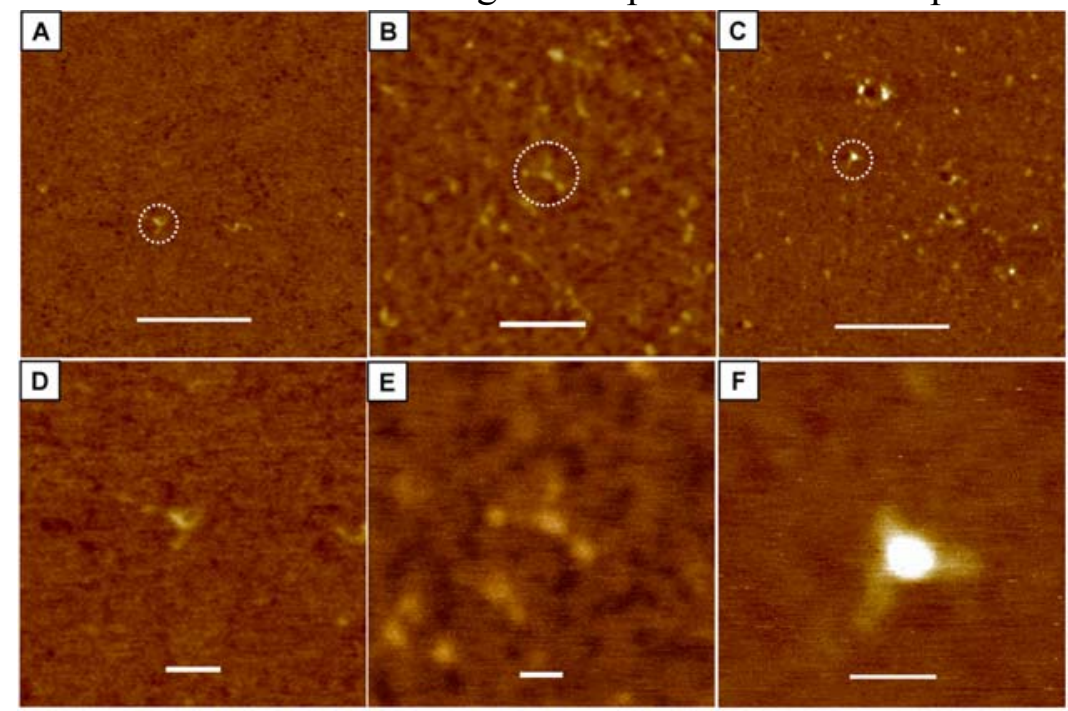

Figure S-1. Representative AFM height images of mica substrates after treatment with three-branched DNA solutions. (A-B) Overview images of surfaces with complex A, showing fully formed structures (circled), as well as incomplete oligonucleotide assembly products; the white bar is $200 \mathrm{~nm}$ in (A) and $100 \mathrm{~nm}$ in (B). (C) Overview image of a surface with complex $\mathbf{B}$, having the completely assembled product (circled), along with incompletely formed and higher-order complexes; scale bar is $200 \mathrm{~nm}$. (D-F) Zoom images of the circled complexes in (A-C); scale bar is $50 \mathrm{~nm}$ in (D) and $20 \mathrm{~nm}$ in (E-F). Height scale is $3 \mathrm{~nm}$ in all images. 


\section{Surface deposition yield}

After deposition typical substrates had an average of $\sim 10$ DNA complexes $/ \mu \mathrm{m}^{2}$, but the density of structures with unambiguously identifiable, separated branches was $\sim 1 / \mu \mathrm{m}^{2}$ for $\mathbf{A}$ and $\sim 0.4 / \mu \mathrm{m}^{2}$ for $\mathbf{B}$ on their respective substrates. Similar surface densities were observed for complexes synthesized under different assembly conditions, indicating that factors other than hybridization efficiency are responsible for the reduced apparent yield of complexes on surfaces. Clear identification of three-branched nanostructures was likely limited by AFM tip-induced broadening and non-coplanar deposition of the three arms of the complexes on the mica surface. Tip broadening reduces the range of angles over which separation of the branches of a flexible (ssDNA core) multi-armed object can be resolved. Likewise, the surface structure, which is a two-dimensional projection of the three-dimensional configuration of the complex in solution, ${ }^{2}$ can result in the three arms not always lying in a coplanar fashion on the mica surface. Because of these factors, the true yield in solution of complexes $\mathbf{A}$ and $\mathbf{B}$ is likely much higher than the apparent yield from AFM images. The surface deposition of three-branched DNAs could be optimized for higher apparent AFM yields by using spin coating, ${ }^{3}$ molecular combing, ${ }^{4-5}$ or gas flow alignment. ${ }^{6}$ Examples of typical AFM data that do not clearly show the expected complex B are shown in Figure S-2.

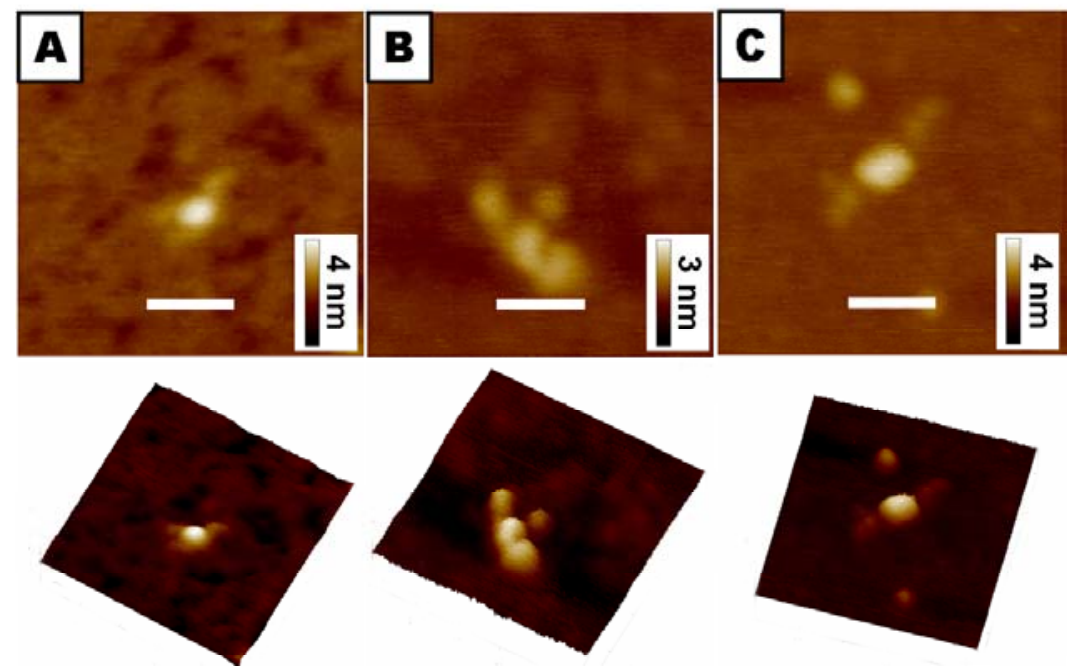

Figure S-2. Tapping-mode AFM height images of surfaces where complex B was deposited. (A) Arms of the complex cannot be resolved clearly; white bar is $40 \mathrm{~nm}$. (B) One arm of the complex appears to be detached; white bar is $25 \mathrm{~nm}$. (C) Incompletely assembled oligonucleotides with conjugated streptavidin; scale bar is $20 \mathrm{~nm}$.

\section{Complex formation yield in solution}

We investigated the solution formation yield of complexes $\mathbf{A}$ and $\mathbf{B}$ by direct injection of the reaction mixtures into a $2 \%$ agarose slab gel. For visualization of the DNA bands, the oligonucleotide concentrations were adjusted so that each well contained $\sim 1 \mu \mathrm{g}$ of DNA, resulting in solutions $\sim 8000$-fold more concentrated than those used for surface deposition. Figure S-3A shows the change in mobility between one oligonucleotide (3: lane 2), two oligonucleotides mixed together $(\mathbf{1}+\mathbf{2}$ : lane 4$)$, and the combination of oligonucleotides 1-3 to form complex A (lane 6). Gel band intensities in lane 6 indicate that $\sim 60 \%$ of the reaction mixture forms complex $\mathbf{A}$, while the remaining $\sim 40 \%$ results in higher molecular weight species. These higher-order complexes may result from the fact that conditions for hybridization were not reoptimized for the higher oligonucleotide concentrations. Figure S-3B depicts the variation in 
mobility during stepwise conversion of complex A (lane 2) into complex B (lane 4). The addition of the biotinylated oligonucleotide (4) to complex $\mathbf{A}$ did not affect the mobility significantly (lane 3). However, the subsequent addition of streptavidin to form complex B significantly reduced the mobility relative to complex $\mathbf{A}$, which was consistent with the $>40 \%$ rise in mass (and considerable increase in hydrodynamic radius). Solution assembly yields for complexes $\mathbf{A}$ and $\mathbf{B}$ from band intensities in several gels were $>50 \%$, with the remainder of the material forming higher molecular weight species, and very little evidence of incomplete hybridization. We also excised the band corresponding to complex A from the gel shown in Figure S-3A with a Qiaquick (Qiagen) DNA extraction kit and used AFM to analyze the DNA complexes deposited on mica surfaces. Three-armed DNA nanostructures were observed (Figure S-4), confirming our identification of this band.

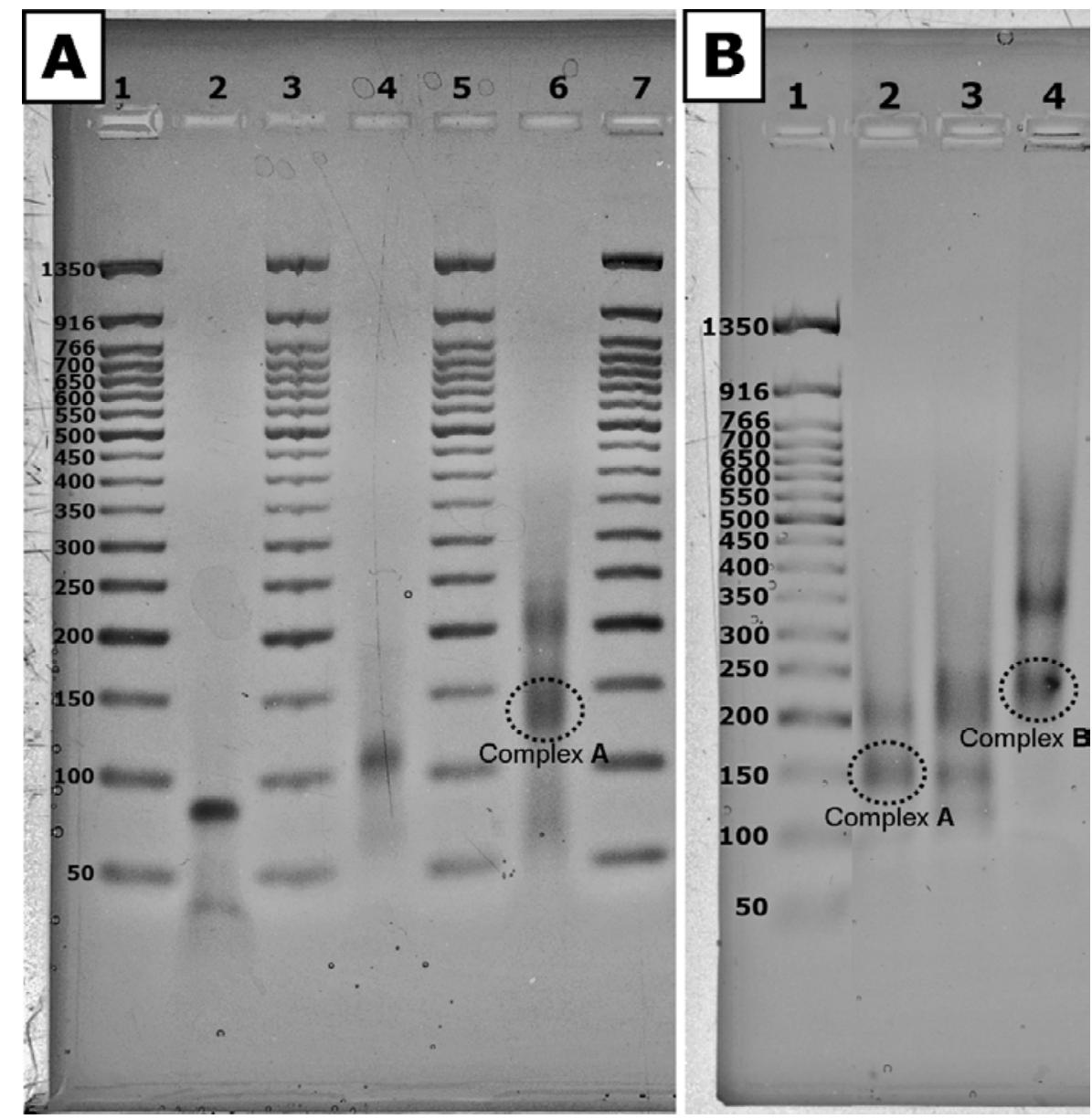

Figure S-3. Gel electrophoresis of branched DNA structures. (A) Formation of complex A. Lanes 1, 3, 5, 7 contained a 50 bp DNA ladder (New England Biolabs, Beverly, MA). Lane 2 contained oligonucleotide 3 and gave a band at $\sim 80 \mathrm{bp}$. Lane 4 contained an equimolar mixture of oligonucleotides 1 and $\mathbf{2}$, resulting in a band at $\sim 110 \mathrm{bp}$. Lane 6 contained an equimolar mixture of oligonucleotides 1, 2 and 3 and gave two bands at $\sim 140$ and $210 \mathrm{bp}$, with relative intensities of $58 \%$ and $42 \%$, respectively. (B) Formation of complex B from complex A. Lane 1 contained a 50 bp DNA ladder. Lane 2 had complex $\mathbf{A}$ and showed the same bands observed in (A), lane 6. Lane 3 contained complex A plus oligonucleotide 4. Lane 4 had added streptavidin to form complex $\mathbf{B}$, and two bands at $\sim 230$ and $330 \mathrm{bp}$ were visible with relative intensities of $62 \%$ and $38 \%$, respectively. 


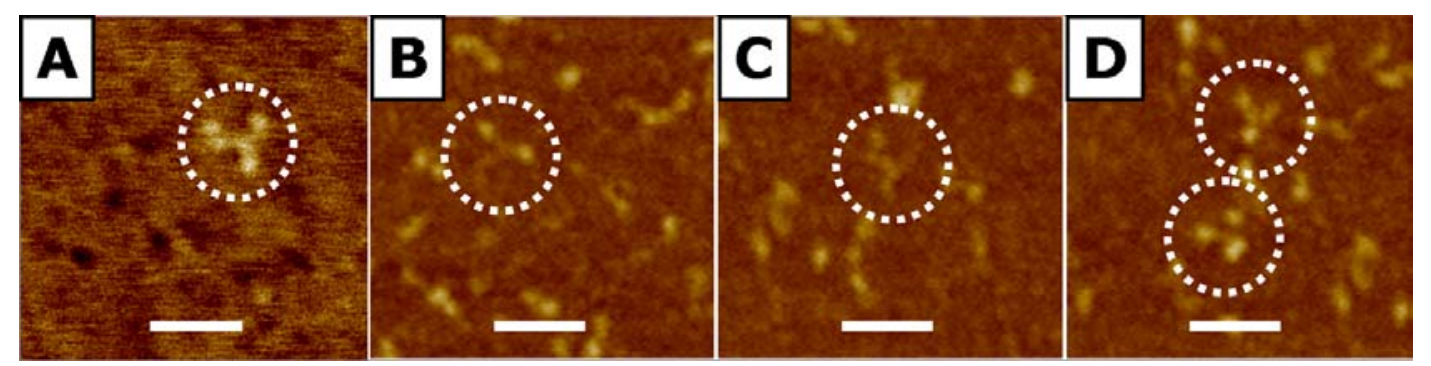

Figure S-4. Representative AFM images of mica-deposited DNA extracted from the $\sim 140$ bp band in Figure S-3A, lane 6. Three-branched structures (complex A) are circled. Scale bar is $50 \mathrm{~nm}$ and height scale is $3 \mathrm{~nm}$ in all images.

\section{Metallization of DNA complexes}

Poly-L-lysine treated carbon-coated copper TEM grids were incubated with $5 \mu \mathrm{L}$ of $\mathbf{A}$ for $3 \mathrm{hr}$ and rinsed. Silver metallization involved treating the grids in the dark for 10 min with an ethanolic solution of $\mathrm{AgNO}_{3}$ (Fisher Scientific, Fair Lawn, NJ) made basic with $\mathrm{NH}_{3}$ (EM Science, Gibbstown, NJ). The grids were rinsed and exposed to a dilute aqueous formaldehyde solution (37\%, Fisher) for 3-20 min. The reaction was terminated by extensive rinsing and air drying. Copper metallization required incubating the grids with a $1 \mathrm{M}$ solution of $\mathrm{Cu}\left(\mathrm{NO}_{3}\right)_{2}$ (Mallinckrodt Baker, Paris, KY) in dimethyl sulfoxide (EM Science) for $10 \mathrm{~min}$. The grids were rinsed and the DNA-associated copper ions were reduced utilizing a $1 \mathrm{M}$ aqueous solution of ascorbic acid (Sigma, St. Louis, MO) for $4 \mathrm{~min}$. The reaction was terminated by rinsing.

\section{Electron microscopy}

TEM images were obtained using a Tecnai 30 FEI instrument, with $300 \mathrm{kV}$ acceleration potential and $3.8 \mathrm{kV}$ extraction voltage. Scanning transmission electron microscopy (STEM) images were recorded on a Tecnai 20 Analytical FEI instrument, with $200 \mathrm{kV}$ acceleration potential and $3.4 \mathrm{kV}$ extraction voltage.

\section{Self assembly reproducibility}

The robustness of our assembly system was verified by direct ultramicroscopy (AFM and TEM) inspection of individual complexes assembled from different reaction mixtures and deposited under various sets of conditions on $\sim 400$ different mica surfaces and $\sim 50$ TEM grid surfaces. While the assembly yield depended on a range of solution and surface condition variables, we observed three-branched DNA complexes on $\sim 65 \%$ of the substrates.

\section{Experimental variability of complex size}

We investigated the apparent size of non-metallized and metallized complexes by tapping-mode AFM and TEM, respectively. The expected arm length of non-metallized complex A or B should be $\sim 21 \mathrm{~nm}$, or slightly more if the ssDNA core is elongated. From AFM images of 40 complexes we measured an average arm length of $26 \mathrm{~nm}$ with a standard deviation of $5 \mathrm{~nm}$ (Figure S-5A). We noticed no statistically significant difference in arm length between complexes $\mathbf{A}$ and $\mathbf{B}$. We also used a model with a $10 \mathrm{~nm}$ assumed tip diameter to calculate that tip-induced broadening would cause an apparent increase in length of the arms of complexes $\mathbf{A}$ and $\mathbf{B}$ of $\sim 4 \mathrm{~nm}$ (Figure $\mathrm{S}-5 \mathrm{~B}$ ); tip and DNA orientation could induce additional variation. We therefore conclude that the observed three-branched structures have dimensions that are consistent with those of complexes $\mathbf{A}$ and $\mathbf{B}$. 

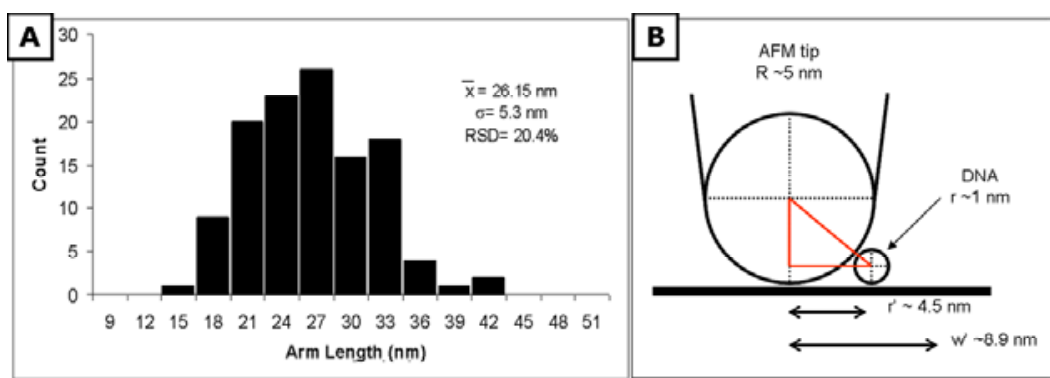

Figure S-5. Analysis of the dimensions of DNA complexes A and B. (A) Arm length histogram for 40 different complexes. (B) Geometric estimation of tip-induced broadening of DNA in AFM imaging.

For metallized complexes we used TEM to measure the length and width of the arms of the resulting structures and found that the arm width of the metallized complexes rapidly ( $<7 \mathrm{~min}$ ) reaches 15-20 nm, approximately the length of the DNA arms. Upon continued metallization, the crystal grows beyond the DNA template, and autocatalytic deposition elongates the distinct branches without much increase in their width. Figure S-6A,B illustrates two DNA-templated silver nanostructures after different plating times, while Figure S-6C,D shows length and width histograms for the arms of 15 silver-metallized complexes prepared like the structure in Figure S-6B; the narrowest metallized arm width we have observed thus far by TEM is $12 \mathrm{~nm}$.

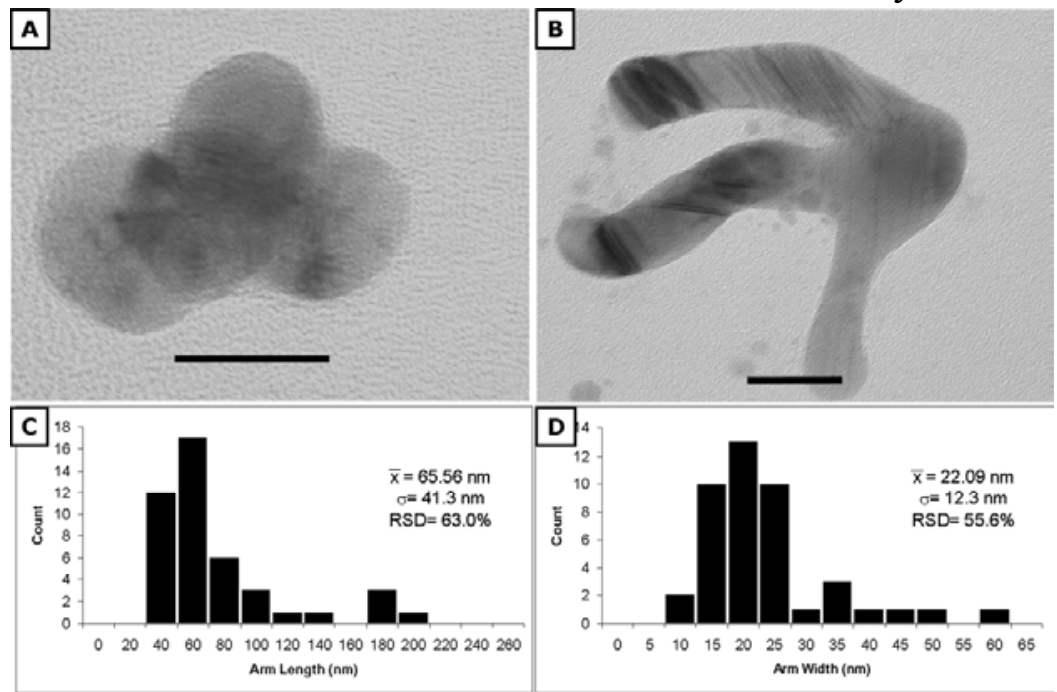

Figure S-6. Analysis of metallized DNA complex A. (A) Silver-metallized product after $\sim 7$ min of plating. (B) A different silver metallization product after $20 \mathrm{~min}$ of treatment. Scale bars are $20 \mathrm{~nm}$ in (A-B). (C) Arm length histogram for 15 different complexes plated for $20 \mathrm{~min}$. (D) Arm width histogram for the same complexes as in (C).

\section{Clustered DNA structures}

Deposition of three-branched DNA complexes on TEM grids resulted in an increased tendency of the DNA molecules to form higher-order structures (Figure 2D,F,G). We attribute the formation of these assemblies to the hydrophobic nature of the carbon-coated TEM grids; this causes the more hydrophilic DNA complexes to cluster. These bundled structures metallize readily, forming characteristic filaments with bifurcations at regular intervals. While it is possible that metallization products of entangled linear DNAs might have superficial similarities to these metallized bundles of branched complexes, it is easy to distinguish them by the regularity of the branching. Moreover, we have also observed limited instances of bundling of three-branched complexes on mica surfaces, and we present two of these in Figure S-7. 

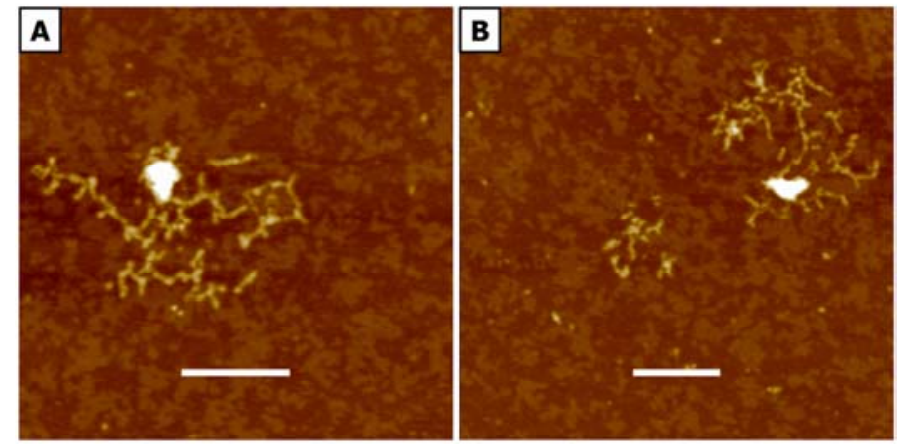

Figure S-7. Tapping-mode AFM height images of regularly bifurcating clusters of three-branched DNA on mica. These bundles were formed in the vicinity of surface contamination. White bars are $200 \mathrm{~nm}$ and height scale is $3 \mathrm{~nm}$ in both images.

\section{EDX analysis}

Silver

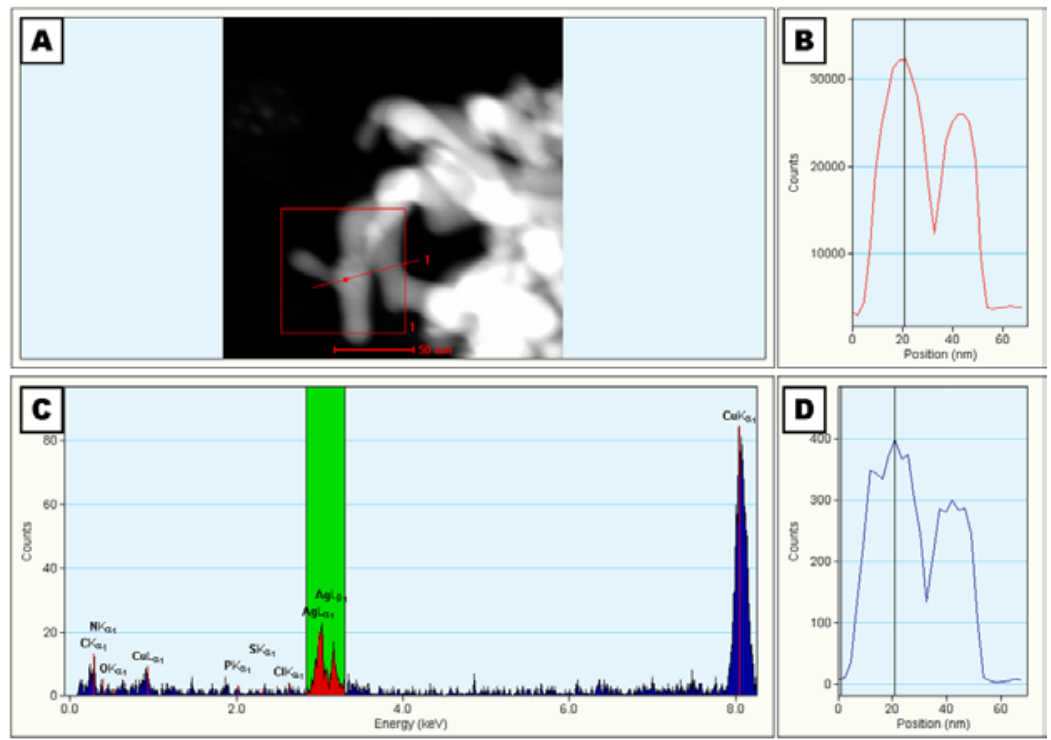

Figure S-8. STEM EDX line profile on a drift-corrected region of a cluster of silver-metallized three-branched DNAs. (A) STEM image, showing the analyzed feature, the region used for Fourier transform drift correction (red box) and the path of the line scan (red line). The small dot along the red line represents a spot in the image, which is analyzed in (B-D). (B) Raw trace of the high-angle annular dark-field detector along the line scan. The black vertical line indicates the position of the dot in (A). (C) X-ray spectrum for the position marked by the dot in (A). Silver, carbon and copper (from the grid) are the main constituents. The green region encompasses the $\mathrm{Ag}_{\mathrm{L}} \mathrm{X}$-rays. (D) Integration of the green region in $(\mathrm{C})$ shows a silver content profile that closely resembles that of the total signal in (B) and is in full registry with the image in (A). 


\section{Copper}
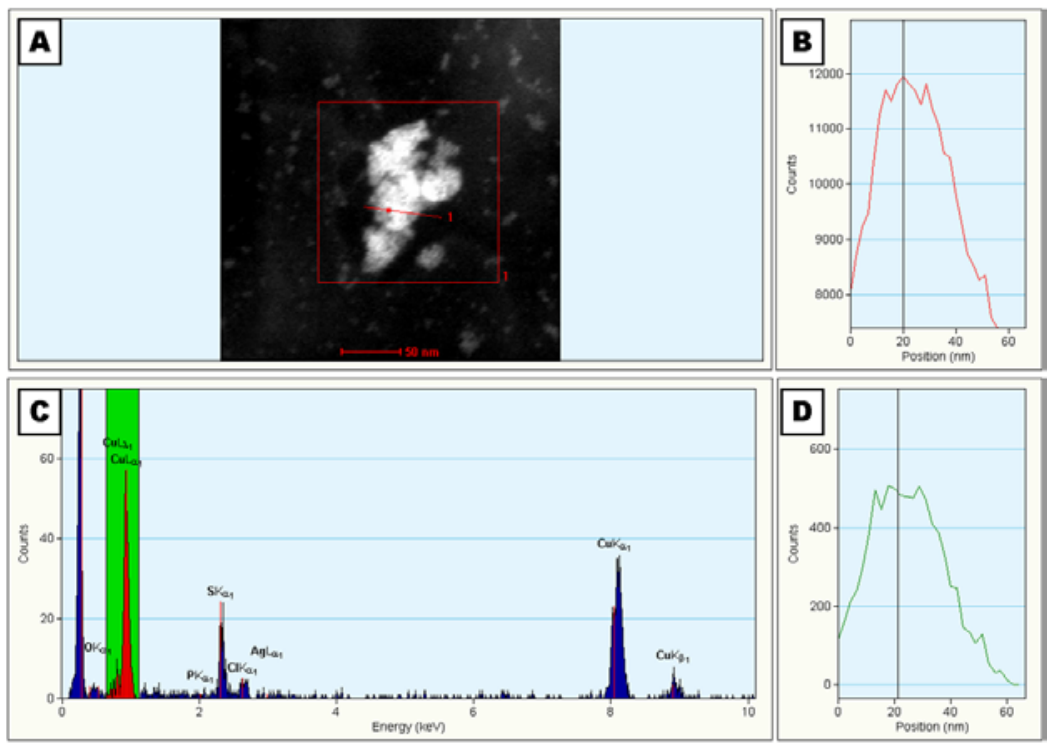

Figure S-9. STEM EDX study of complex A metallized with copper. (A) STEM image with drift correction region (red box) and line scan path (red line) indicated. The dot on the red line represents a spot in the image, which is analyzed in (B-D). (B) Total detector signal traces the shape of (A). The black line corresponds to the red dot in (A). (C) EDX spectrum at the point indicated by the red dot in (A); the green region encompasses the $\mathrm{Cu}_{\mathrm{L}} \mathrm{X}$-rays. (D) Profile of the integrated $\mathrm{Cu}_{\mathrm{L}}$ signal from the green-shaded area in (C) along the line scan.

\section{References}

${ }^{1}$ Mathews, D. H.; Zucker, M.; Turner, D.H. RNAstructure, ver. 3.7, University of Rochester, New York, 2002.

${ }^{2}$ Varadhan, G.; Robinett, W.; Erie, D.; Taylor, R. M., II. In Visualization and Data Analysis, Erbacher, R. F.; Chen, P. C.; Groehn, M.; Roberts, J. C.; Wittenbrink, C. M., Eds. Proc. SPIE: Bellingham WA, 2002; Vol. 4665, pp 116124.

${ }^{3}$ Harnack, O.; Ford, W. E.; Yasuda, A.; Wessels, J. M. Nano Lett. 2002, 2, 919-923.

${ }^{4}$ Bensimon, A.; Simon, A.; Chiffaudel, A.; Croquette, V.; Heslot, F.; Bensimon, D. Science 1994, $265,2096-2098$.

${ }^{5}$ Woolley, A. T.; Kelly, R. T. Nano Lett. 2001, 1, 345-348.

${ }^{6}$ Xin, H.; Woolley, A. T. Nano Lett. 2004, 4, 1481-1484. 